\title{
Marfan's syndrome. Early and Severe Form in Siblings
}

\author{
Maria de Fátima M. P. Leite, Nadia Barreto Tenorio Aoun, Monica Scott Borges, \\ Maria Eliane Campos Magalhães, Luiz A. Christiani
}

Rio de Janeiro, RJ - Brazil

\begin{abstract}
Marfan's syndrome is an inherited disorder of the connective tissue. Cardiologic manifestations, especially aortic dilation, are important causes of morbidity and mortality in the clinical course of the disease in adults and teenagers. In children, the presence of aortic aneurysm and its dissection or rupture is rare, occurring in patients with genetic mutation of the fibrillin gene but not in those who have the familial form of the disease. We describe here 2 patients, from the same family (siblings), diagnosed with gigantic aortic aneurysm early in infancy, one of them successfully undergoing surgery.
\end{abstract}

Marfan's syndrome is an autosomal dominant inherited disorder of the connective tissue-with multisystemic involvement, caused by mutations in the locus of the fibrillin gene on chromosome $15^{1}$. Its prevalence is $1 / 10,000$ inhabitants in the general population, with no preference regarding ethnicity, sex, or race. It is the most common inherited disorder of the connective tissue ${ }^{1}$. Most characteristic alterations are cardiovascular, skeletal, and ocular ${ }^{1}$. Cardiovascular alterations, especially aortic aneurysm, are the most severe manifestation of the disease, especially in adults. Mortality due either to dissection or rupture is high, reaching $50 \%$ if not treated ${ }^{2,3}$. Important cardiovascular alterations during childhood are rare, especially among members of the same family, and few studies on this subject have been published ${ }^{3,4}$. The mean age of the onset of symptoms is the second or third decade of life, once the disease has progressed, especially regarding aortic alterations ${ }^{3}$. We present here 2 cases of Marfan's syndrome with severe and early cardiovascular manifestation in patients from the samefamily.

Hospital Universitário Pedro Ernesto - Universidade do Estado do Rio de Janeiro. Mailing address: Maria de Fátima M. P. Leite - Rua Magalhães Couto, 784 - Casa 34 - 20735-180 - Rio de Janeiro, RJ - E-mail: nbtaoune@ cardiol.br

\section{Cases report}

Case 1 - A 9-year-old nonwhite female child, born in Rio de Janeiro, was sent to Hospital Universitário Pedro Ernesto (HUPE-UERJ) (Pedro Ernesto University Hospital), for treatment of a cardiac murmur. The child was born from a normal delivery at term, weighting 2,600g. Her motor development was retarded (she sat up without support at 2 years of age and walked at 4 years). Her psychological development was normal. She experienced repetitive pneumonias from the 6th month of life. The mother was healthy, the father and 2 brothers ( 10 and 12 years old) had mitral valve prolapse, and 1 brother ( 8 years old) had clinical manifestations similar to those of the patient.

A physical examination revealed the following: weight $20 \mathrm{~kg}$; height $1.20 \mathrm{~m}$; atypical facies; normal skin; measurement of the hands showed arachnodactyly; a high arched palate; anterior chest deformity at the level of the 7th and 9th right ribs (fig. 1). An ophthalmology examination was normal. A precordial bulge was present on the sternal furcula. The patient had a slow pulse, apical thrust in the 6th left intercostal space of 2 fingerbreadths, her 2 nd heart sound was palpable, cardiac rhythm was regular, without accessory sounds. An accentuated sound $\left(A_{2}\right)$ was present as were an aortic ejection click, a systolic murmur $++/ 6$ in the accessory aortic area, and a short diastolic murmur $++/ 6$ in the aortic area. Her blood pressure was $100 / 50 \mathrm{mmHg}$, and heart rate was $100 \mathrm{bpm}$. She also had a bilateral inguinal hernia, and an osteoarticular examination showed joint hypermobility in the ankles, knees, rips, scapula, and flat feet (pes planus). A chest $X$-ray showed cardiomegaly with mediastinum deviation to the left, and important dilation of the ascending aorta. An ECG showed atrial and ventricular enlargement and 1st degree left bundle-branch block. An echocardiogram revealed left atrium and ventricle enlargement, an aortic aneurysm measuring $6.0 \mathrm{~cm}$ in diameter, and mild aortic regurgitation (fig. 2). A hemodynamic study revealed these pressures: AO: 140/59 (118) $\mathrm{mmHg}$ and VE: $140 / 0 / 18 \mathrm{mmHg}$. Angiography showed left ventricle enlargement, an ascending aortic aneurysm with a root diameter 


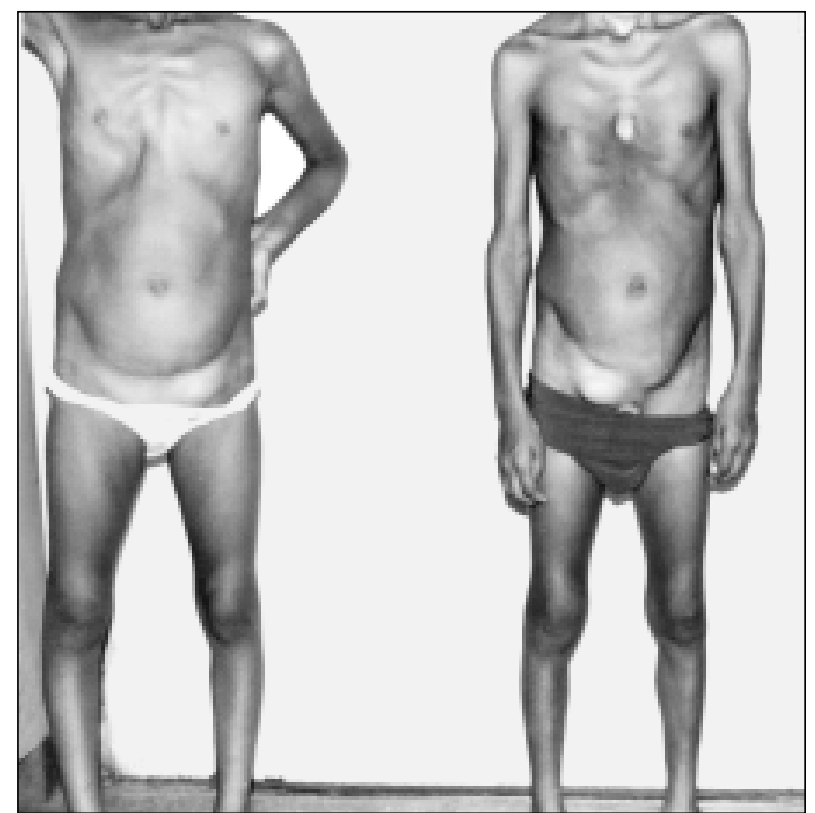

Fig. 1 - Picture 1 showing thoracic alterations and the patients' inguinal hernia.

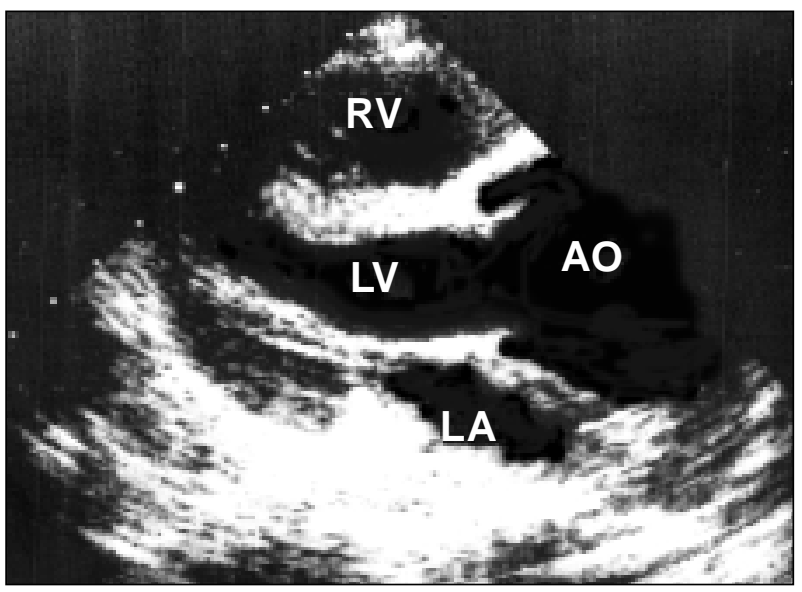

Fig. 2 - Longitudinal cut echocardiogram. Bulky aortic aneurysm in patient 1.

measuring $6.0 \mathrm{~cm}$, and mild aortic regurgitation. A genetic investigation evaluated mutations of fibrillin gene; however, on this occasion, the examination was not performed in Brazil, and the sample sent for analysis was contaminated; therefore, -genetic analysis was not possible.

We started treatment with propranolol $1 \mathrm{mg} / \mathrm{kg} /$ day and indicated surgical correction. At the preoperative appointment, 1 month after surgical indication, the patient was admitted to our service presenting with precordialgia, dyspnea, tachycardia, and sweating. Aortic dissection was diagnosed. The patient was indicated for emergency surgery to place a mechanical prostheses in the aortic position and a Dacron graft in the ascending aorta. Unfortunately, we did not perform an anatomopathologic study. After surgery, she evolved with systemic hypertension that was controlled with angiotensin-converting enzyme inhibitors and atenolol. We started anticoagulation with Cumarin because of the mechanical prostheses. After 4 years of follow-up, receiving only atenolol and anticoagulation therapy, the child abandoned treatment evolving to death in 1 year.

Case 2 - An eight-year-old nonwhite male child, born in Rio de Janeiro, was sent to HUPE with the same complaints as those of his sister (case 1). He was born from a Cesarean delivery due to fetal distress, weighting 2,200g. His familial and physiological history were similar to that of case 1 , including an important delay in motor development (he walked at 4 years). He experienced repetitive pneumonia and bilateral inguinal herniorrhaphy at 2 years. A physical examination revealed the following: weight $17 \mathrm{~kg}$, height $1.18 \mathrm{~m}$, facies atypical, normal skin hypertelorism, a high arcade palate, hands suggestive of arachnodactyly, and pectus excavatum (fig. 1). The ophthalmology examination was normal. His heart beat was palpable at the sternal furcula. He had a slow pulse; a precordial bulge at the 6th left intercostal space, 2 fingerbreadths; palpable $\mathrm{A}_{2}$; regular rhythm; an accentuated 2 nd heart sound $\left(\mathrm{A}_{2}\right)$; an aortic ejection click, a systolic murmur $++/ 6$ in the accessory aortic area; a diastolic murmur $+/ 6$ in the aortic area; and blood pressure of $100 / 50 \mathrm{mmHg}$. He also had an incisional hernia in the bilateral inguinal region. An osteoarticular examination showed joint hypermobility in the knees and scapula and flat feet. A chest X-ray showed pectus excavatum type chest alterations, the mediastinum deviated to the left, and cardiomegaly. The ECG showed 1st degree left bundlebranch block. An echocardiogram showed left atrium and ventricle enlargement, an aortic aneurysm measuring $6.5 \mathrm{~cm}$ in diameter, mild to moderate aortic regurgitation, and left ventricle hyperkinesias. The hemodynamic study revealed a pressure of $\mathrm{AO}=125 / 60(87) \mathrm{mmHg}$, and angiography demonstrated an aortic aneurysm, with mild to moderate aortic regurgitation (fig. 3). A genetic evaluation was hindered by contamination of the sample.

Propranolol $1 \mathrm{mg} / \mathrm{kg} /$ day was started; however, the patient had bacterial meningitis and was admitted to another hospital where he died.

\section{Discussion}

The most frequent cardiovascular alterations in Marfan's syndrome are mitral valve prolapse, which may cause significant regurgitation, and progressive dilation of the aortic valvar annulus, leading to dissection or rupture of this vessel, being the main cause of morbidity and mortality in these disorders. In some studies ${ }^{3}$, morbidity occur in $97 \%$ of patients, and when treatment is not performed, $50 \%$ die around the 4 th decade of life ${ }^{3}$ due to some cardiovascular complication. Rupture or aortic aneurysm dissections are the most frequent complications ${ }^{2}$.

Echocardiographic studies in children have demonstrated that aortic root dilation is the most frequent cardiovascular alteration after mitral valve prolapse ${ }^{3}$, and that aortic regurgitation is, in the majority of cases, a consequence of the progression of ascending aortic dilation that occurs later in life. However, cases exist where severe aortic 


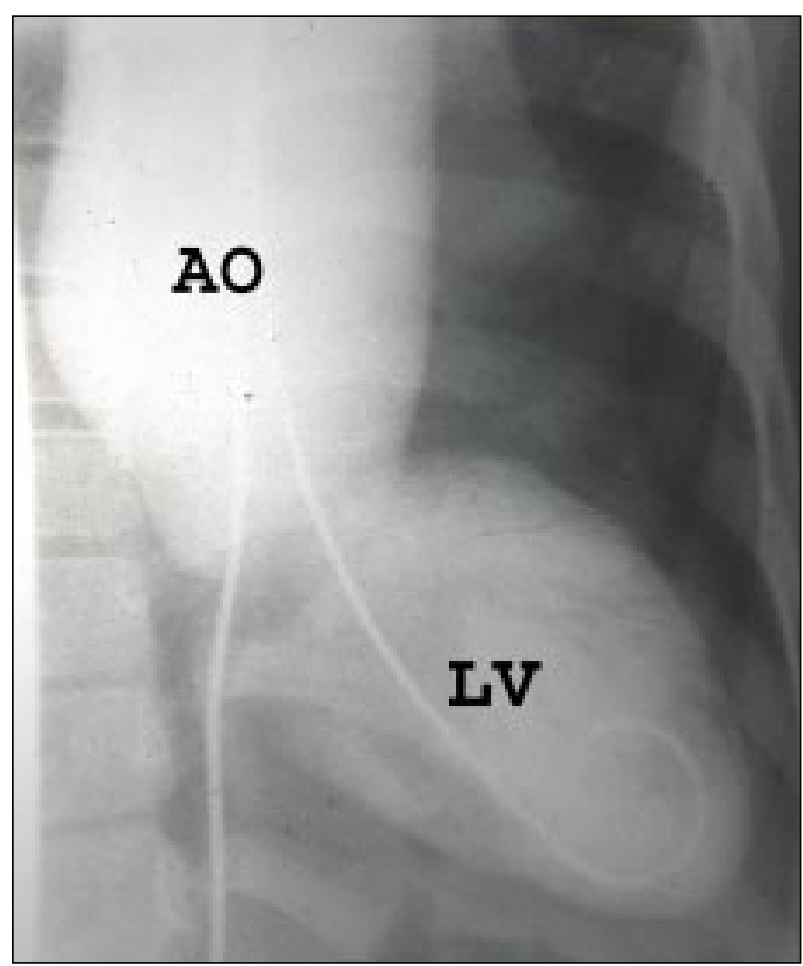

Fig. 3 - Patient 2's ventriculography, demonstrating a bulky aneurysm that involves the ascending aorta.

dilation occurs before adolescence, with the presence of aneurysms, and these patients are considered to have a severe form of the disease ${ }^{5}$.

During the neonatal period and childhood, clinical manifestation of Marfan's syndrome is of minor significan$\mathrm{ce}^{6}$, and patients remain asymptomatic until the third decade of life, when diagnosis is most commonly made. In general, before this period, a diagnosis is only made in patients with the most severe form of the disease with early manifestations of heart failure, or in the presence of cardiac murmurs suggestive of mitral valve prolapse or aortic regurgitation, or also in the routine examination of patients with a family history. Our patients had severe aortic dilation with mild to moderate regurgitation, and one of them had aortic dissection, which is unusual at that age ${ }^{3,5,7}$. Another interesting fact is the family history, because, generally, most severe and early cases of the disease are those that occur in isolation $^{2}$. Our patients were not only from the same family but also had a father and a brother with mitral valve prolapse, without the other phenotypic characteristics of Marfan's syndrome.

When Marfan's syndrome is suggested, patients and their family must be thoroughly investigated, to confirm the disease, and to initiate the treatment, thus decreasing mortality, especially due to cardiovascular causes. The use of beta-blockers in aortic dilation is considered essential nowadays, because it seems to slow down the progression of the disease, therefore, delaying the need for surgery ${ }^{7}$. For adequate follow-up, echocardiography is of utmost importance, because it is a noninvasive method that is easy to perform, and good resolution that enables diagnosis and follow-up of both mild alterations (small aortic dilations, mitral valve prolapse), and severe ones (gigantic aneurys$\mathrm{ms}$, severe mitral malformations). Other methods of diagnosis, such as hemodynamic study, magnetic resonance, and tomography, must be used wisely. In the present study, echocardiographic examination was decisive in the evaluation of the patients, and the angiographic evaluation helped in the quantification of aortic regurgitation and of the extension of the dilated area. An excellent correlation occurred between the 2 methods.

Regarding clinical treatment of aortic aneurysms, medications like reserpine, trimetaphan, and guanethidine started being used in $1950^{7}$. In the $1960 \mathrm{~s}^{7}$, propranolol was used for treating long-term follow-up patients with aortic dilation, avoiding its progressive evolution. In adults, the drug seems to obtain the expected effects ${ }^{8}$; however, in children, no definitive evidence exists of a decrease in morbidity and mortality ${ }^{6}$. Because the results in adults are promising, the use of beta-blockers is mandatory in all patients with aortic root dilation, even for pediatric patients. Our patients started propranolol use $1 \mathrm{mg} / \mathrm{kg} /$ day just after the diagnosis of aortic root dilation and maintained its use until surgery and, even so, one case of aortic dissection occurred (case 1).

Surgical treatment is indicated, electively in patients with bulky aneurysms measuring from 5.5 to $6.0 \mathrm{~cm}^{2,3}$ in diameter, even in those asymptomatic patients, because of the great risk of rupture and dissection, increasing the need emergency surgery ${ }^{3}$. Postoperative mortality for aortic aneurysms is decreasing in patients with Marfan's syndrome. Some decades ago, it was around $40 \%$. Currently preoperative mortality for elective surgery of aortic aneurysms is lower than $2 \%{ }^{9}$. The mortality rate for patients who undergo emergency surgical intervention due to acute aortic dissection, or aneurysm rupture, however, is 8 times greater ${ }^{9}$. In children, a survival of $70 \% \pm 10$ in 10 years is statistically observed ${ }^{9}$. One of the main causes of morbidity is the need for reoperation when distal aortic disease occurs, or in the presence of aortic root dilation after mitral valve surgery. In adults, the main indication for reoperation is aortic dissection ${ }^{5}$. In addition to these, infectious endocarditis is also an important cause of morbidity and mortality ${ }^{10}$.

Case 1 was a patient who underwent emergency surgery successfully, with placement of a Dacron graft and aortic mechanical prostheses. She was followed up for 4 years and died because she abandoned treatment and suspended anticoagulation therapy after this period. Patient 2 died before surgery because of noncardiac events.

It is believed that disorders in the glycoprotein fibrillin are responsible for the cardiac, ocular, and skeletal alterations of Marfan's syndrome ${ }^{5}$. These alterations may be familial or may be a consequence of genetic mutations; therefore, genetic counseling is essential in Marfan's syndrome. Parents of patients with Marfan's syndrome must be investigated for the familial form of the disease or for evidence of genetic mutation in the affected child, so that they 
can be guided regarding the risk of the disease in other members of the family. Women with the syndrome should be told the risks of pregnancy, because they can experience complications resulting from cardiac disease, such as aortic aneurysm rupture ${ }^{10}$ or infectious endocarditis ${ }^{10}$ during deli- very or puerperium. These patients experience a greater number of miscarriages compared with healthy women ${ }^{10}$. With the advent of genetic therapy, we hope the precocious approach to Marfan's syndrome results in an even better treatment for this ailment in the future.

\section{References}

1. Fleisher KJ, Nousari HC, Anhalt GJ, Stone CD, Laschinger JC. Immunohistochemical abnormalities of fibrilin in cardiovascular tissues in Marfan's syndrome. Ann Thorac Surg 1997; 63: 1012-7.

2. Yoshinaga M, Oku S, Nagai E. A case of Marfan syndrome in an adolescent with aortic dissection: should the recommendation for surgical intervention be changed? Ped Cardiol 1999; 20: 431-4.

3. Gillinov AM, Zeher KJ, Redmond JM, et al. Cardiac operations in children with Marfan's syndrome: indications and results. Ann Thorac surg 1997; 64: 1140-5.

4. LababidiZ, Monzon C. Early cardiac manifestations of Marfan's syndrome in the newborn. Am Heart J 1981; 102: 943-5.

5. Devereux RB, Roman MJ. Aortic disease in Marfan's syndrome. N Eng J Med 1999; 340: 1358-9.
6. Morse RP, Rockenmacher S, Pyeritz RE, et al. Diagnosis and management of infantile Marfan syndrome. Pediatrics 1990; 86: 888-94.

7. Kornbluth M, Schnittger I, Eyngorina I, Gasner C, Liang DH. Clinical outcome in the Marfan syndrome with ascending aortic dialtation followed annually by echocardiography. Am J Cardiol 1999; 84: 753-5.

8. Groenink M, de Roos A, Mulder BJ, Spaan JA, van de Wall EE. Changes in aortic distensibility and pulse wave velocity assessed with magnetic resonance imaging following beta-blocker therapy in the Marfan syndrome. Am J Cardiol 1988; 82: 203-8.

9. Gott VL, Greene PS, Alejo DE, et al. Replacement of the aortic root in patients with Marfan's syndrome. N Engl J Med. 1999; 340: 1307-13.

10. Ferguson JE, Ueland K, Stinson EB, Maly RP. Marfan's syndrome: acute aortic dissection resulting in fetal distress and cesarean section, followed by successful surgical repair. Am J Obstet Gynecol 1983; 7: 759-62. 